\title{
QUADRUPOLE INTERACTIONS AND ANISOTROPIC MAGNETIC QUENCHING OF POSITRONIUM IN ORIENTED CRYSTALS
}

\author{
I.V. Bondarev aNd S.A. Kuten
}

Institute of Nuclear Problems at Belarussian State University Bobruiskaya St. 11, 220050 Minsk, Republic of Belarus, CIS

The quadrupole interaction of positronium in noncubic defectless crystals has been investigated theoretically. It is shown to lead to the weak anisotropy of positronium magnetic quenching. The temperature dependence of the quadrupole coupling constant and the estimations of the magnitude of the anisotropy are obtained for delocalized positronium in crystalline quartz. The significant magnitude of the anisotropy is predicted for "anomalous" positronium in oriented crystalline polymeric structures.

PACS numbers: 31.30.Gs, 36.10.Dr, 71.60.+z, 78.70.Bj

\section{Introduction}

It is well known [1] that, due to the noncentral part of the hyperfine interaction between the electron and the nucleus, all hydrogen-like atoms possess quadrupole moments $Q$ in their ground states. For example, $Q$ is equal to 0.7 barn for hydrogen (II), 0.2 barn for deuterium (D), and 2.4 barn for muonium (Mu). Investigation of the quadrupole interactions of H-like atoms in a medium permits not only to study the intracrystalline fields but also the diffusion mechanism of light interstitials in a crystal and the isotope effects [2]. Positronium (Ps) is the lightest H-like atom which at present has been registered experimentally in semiconductors, ionic and molecular crystals, liquids, gases [3-5]. In that connection it is interesting to study the question about the existence of its quadrupole moment in a medium. The point is that, due to the equality of the electron and the positron masses and also due to the execution of the $C P$ - and $T$-invariance laws in the system "particle-antiparticle", free Ps in vacuum is a completely electroneutral system and cannot have any multipole moments [6]. In crystals, however, we deal with a quasi-Ps exciton-like state [3]. In such a state the effective masses of the electron and the positron are, generally speaking, not the same. As a consequence, positronium in a crystal may possess a new fundamental characteristic an effective quadrupole moment [7].

The existence of the effective quadrupole moment in the ground state of Ps, as well as in the case of $\mathrm{Mu}$ and $\mathrm{H}$ [2] would lead to the anisotropic hyperfine interaction between the electron and the positron of the type [8]:

$$
H=A_{i k} S_{\mathrm{e}}^{i} S_{\mathrm{p}}^{k}
$$


with (atomic units)

$$
A_{i k}=\omega \delta_{i k}+Q_{\mathrm{Ps}} \varphi_{i k}
$$

where $A_{i k}$ is the matrix of the hyperfine interaction, $S_{\mathrm{e}}$ and $\boldsymbol{S}_{\mathrm{p}}$ are the spin operators of the electron and the positron, respectively, $\omega$ is the hyperfine splitting of the ground level of Ps in the crystal (in vacuum it is equal to $\omega_{0}=$ $\left.8.2 \times 10^{-4} \mathrm{eV}[6]\right), Q_{\mathrm{Ps}_{\mathrm{s}}}$ is the effective quadrupole moment of $P s$ and $\varphi_{i k}$ is the electric field gradient (EFG) tensor in Ps centre-of-mass.

The purpose of this paper is to analyze theoretically the consequences of Ps effective quadrupole interaction and to obtain the approximate estimations of the magnitude of the effects observed. We also generalize our conclusions to the "anomalous" Ps which was recently detected in crystalline polymers [9].

\section{Possible mechanism of the effective quadrupole moment formation}

The Hamiltonian of the positron-electron bound pair (ground state) in a crystal, with allowance made for the hyperfine interaction between the electron and the positron, can be written in the form (atomic units):

$$
H=-\frac{1}{2}\left(\Delta_{\mathrm{p}}+\Delta_{\mathrm{e}}\right)-\frac{1}{\left|r_{\mathrm{p}}-r_{\mathrm{e}}\right|}+H_{\mathrm{hf}}+V^{(\mathrm{p})}\left(r_{\mathrm{p}}\right)-V^{(\mathrm{e})}\left(r_{\mathrm{e}}\right)
$$

The first two terms in (2) describe free Ps atom in its ground state. The third one is the hyperfine interaction between the electron and the positron [6]:

$$
\begin{aligned}
H_{\mathrm{hf}} & =2 \pi \mu_{\mathrm{e}} \mu_{\mathrm{p}}\left[3+\frac{28}{3} S_{\mathrm{e}} \cdot S_{\mathrm{p}} \delta\left(r_{\mathrm{p}}-r_{\mathrm{e}}\right)\right] \\
& +\frac{4 \mu_{\mathrm{e}} \mu_{\mathrm{p}}}{\left|r_{\mathrm{p}}-r_{\mathrm{e}}\right|^{3}}\left(3 S_{\mathrm{e}} \cdot n S_{\mathrm{p}} \cdot n-S_{\mathrm{e}} \cdot S_{\mathrm{p}}\right)
\end{aligned}
$$

(here, as usual, $r_{\mathrm{e}}, \mu_{\mathrm{e}}, \boldsymbol{S}_{\mathrm{e}}$ and $r_{\mathrm{p}}, \mu_{\mathrm{p}}, \boldsymbol{S}_{\mathrm{p}}$ are the coordinates, the magnetic moments and the spin operators of the electron and the positron, respectively, $\left.n=\left(r_{\mathrm{p}}-r_{\mathrm{e}}\right) /\left|r_{\mathrm{p}}-r_{\mathrm{e}}\right|\right)$. The last two terms describe the interaction of Ps with periodical field of a crystal. We take into account the difference between the electronand the positron-crystal field interaction. This difference comes from the indistinguishability of Ps electron and the electrons of a crystal and provides $V^{(\mathrm{e})} \neq V^{(\mathrm{p})}$.

Using the multipole expansion of the periodical crystalline potential and taking into account the spherically symmetric $(l=0)$ part of the expansion by means of the effective mass technique, we obtain from (2) the Hamiltonian for large radius $\mathrm{Ps}$ exciton [10] in anisotropic $(l \neq 0)$ periodical potential. In the centre-of-mass system of an exciton it is written as*

$$
\begin{aligned}
H= & -\frac{1}{2 M} \Delta_{\boldsymbol{R}}-\frac{1}{2 m} \Delta_{r}-\frac{1}{\varepsilon r}+H_{\mathrm{hf}}(r) \\
& +\sum_{l=1}^{\infty}\left[V_{l}^{(\mathrm{p})}\left(\boldsymbol{R}+\boldsymbol{R}_{\mathrm{p}}\right)-V_{l}^{(\mathrm{e})}\left(\boldsymbol{R}-\boldsymbol{R}_{\mathrm{e}}\right)\right]
\end{aligned}
$$

with

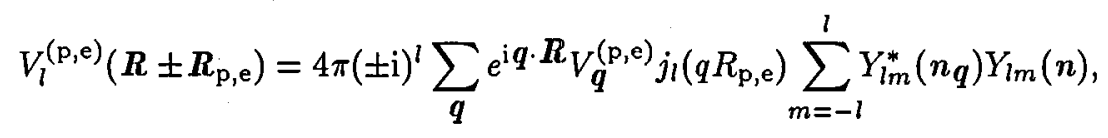

${ }^{*}$ By introducing the coordinate-dependent effective masses and dielectric permeability, the model can be easily generalized to the case of a small radius Ps exciton [11]. 
where $\boldsymbol{R}=\left(\boldsymbol{r}_{\mathrm{e}} m_{\mathrm{e}}+r_{\mathrm{p}} m_{\mathrm{p}}\right) /\left(m_{\mathrm{e}}+m_{\mathrm{p}}\right), \boldsymbol{R}_{\mathrm{e}}=\left(m / m_{\mathrm{e}}\right) \boldsymbol{r}, \boldsymbol{R}_{\mathrm{p}}=\left(m / m_{\mathrm{p}}\right) r, \boldsymbol{r}=\boldsymbol{r}_{\mathrm{p}}-\boldsymbol{r}_{\mathrm{e}}$, $m=m_{\mathrm{e}} m_{\mathrm{p}} /\left(m_{\mathrm{e}}+m_{\mathrm{p}}\right), M=m_{\mathrm{e}}+m_{\mathrm{p}}, m_{\mathrm{e}}$ and $m_{\mathrm{p}}$ are the scalar excitonic effective masses of the electron and the positron, respectively. They are obviously not equal to each other due to the fact that $V^{(\mathrm{e})} \neq V^{(\mathrm{p})}$. In Eqs. (4)-(5) $\varepsilon$ is the optical dielectric permeability, $V_{q}^{(\mathrm{p}, \mathrm{e})}$ - the Fourier transform of the periodical crystalline potential ( $q$ is the reciprocal vector), $j_{l}$ - the spherical Bessel function, $Y_{l m}-$ the spherical harmonics.

If we now assume the positronium-crystal field interaction to be small, then, defining the wave function of our system as $\Psi(\boldsymbol{R}, r)=\psi(r, \boldsymbol{R}) \varphi(\boldsymbol{R})$, we can separate the relative motion and that of the centre-of-mass in (4) [3]. We obtain then

$$
\begin{aligned}
& \left\{-\frac{1}{2 m} \Delta_{\boldsymbol{r}}-\frac{1}{\varepsilon r}+H_{\mathrm{hr}}(\boldsymbol{r})+\sum_{l=1}^{\infty}\left[V_{l}^{(\mathrm{p})}\left(\boldsymbol{R}+\boldsymbol{R}_{\mathrm{p}}\right)-V_{l}^{(\mathrm{e})}\left(\boldsymbol{R}-\boldsymbol{R}_{\mathrm{e}}\right)\right]\right\} \psi(\boldsymbol{r}, \boldsymbol{R}) \\
& =E(\boldsymbol{R}) \psi(r, \boldsymbol{r}),
\end{aligned}
$$

where the energy levels $E(\boldsymbol{R})$ depend parametrically on the centre-of-mass coordinate of Ps exciton and satisfy the equation

$$
\left(-\frac{1}{2 M} \Delta_{\boldsymbol{R}}+E(\boldsymbol{R})\right) \varphi(\boldsymbol{R})=E \varphi(\boldsymbol{R}) .
$$

Under the assumption above, Eq. (6) may be solved by means of the perturbation theory. In doing so, it is convenient to relate the hyperfine interaction to the unperturbed part of the Hamiltonian. Then, due to the noncentral part of $H_{\mathrm{hf}}(r)$ (Eq. (3)), the exact wave function of Ps exciton in the triplet ground state takes the form [1]:

$$
\begin{aligned}
& \psi_{0}^{F=1, m_{F}}(r, \boldsymbol{R})= \\
& \quad R_{S}(r, \boldsymbol{R})\left(Y_{0}(\boldsymbol{n}) \otimes \chi_{1}\right)^{F=1, m_{F}}+R_{D}(r, \boldsymbol{R})\left(Y_{2}(n) \otimes \chi_{1}\right)^{F=1, m_{F}},
\end{aligned}
$$

where $m_{F}$ is the projection of the total angular moment $F=1$ of positronium, $R_{S}(r, \boldsymbol{R}), R_{D}(r, \boldsymbol{R}), Y_{0}(n), Y_{2}(\boldsymbol{n}), \chi_{1}$ are the radial parts, the spherical harmonics of rank 0 and 2 and the spin part (total spin $S=1$ ) of the wave function, respectively. The radial functions $R_{S}(r, R)$ and $R_{D}(r, R)$ describe $S$ - and $D$-wave contributions to the unperturbed ground state (at fixed $R$ ) and characterize the quasi-Ps excitonic state in a medium. They are not identical to those for free Ps atom. This disagreement comes from the electron and the positron effective excitonic masses, from Coulomb potential modified by factor $\varepsilon$, on the one hand, and from the contact part of the hyperfine interaction (3), on the other.

The first order correction to the energy $E(\boldsymbol{R})$ is determined by the diagonal matrix element of the perturbation operator (5). If the hyperfine interaction is taken into account to the first order in small parameter $\mu_{\mathrm{e}} \mu_{\mathrm{p}}$, there is evidently only one, quadrupole, $(l=2)$-term left in the multipole expansion (5) upon the averaging of it over the unperturbed wave function (8). This term can be rewritten in the form

$$
H_{\mathrm{Q}}(\boldsymbol{R})=\frac{Q_{\mathbf{P}_{\mathbf{s}}}(\boldsymbol{R})}{4} Q_{i k} \varphi_{i k}(\boldsymbol{R}),
$$

where $Q_{i k}=F_{i} F_{k}+F_{k} F_{i}-(2 / 3) F(F+1) \delta_{i k}$ is the symmetric Cartesian tensor of rank 2 with zeroth trace, $F=S_{\mathrm{e}}+S_{\mathrm{p}}$ - the total angular moment operator, 
$Q_{\mathrm{P}_{\mathrm{s}}}(\boldsymbol{R})$ - the effective quadrupole moment in the triplet ground state of Ps exciton at fixed position of its centre-of-mass. The atomic quadrupole moment is defined by averaging the quadrupole moment operator in the centre-of-mass system of an atom over th corresponding atomic state with the maximum total moment projection [12]. In our case we have

$$
Q_{\mathrm{Ps}}(\boldsymbol{R})=\frac{m_{\mathrm{e}}-m_{\mathrm{p}}}{m_{\mathrm{e}}+m_{\mathrm{p}}}\left\langle\psi_{0}^{F=1, m_{F}=1}(r, \boldsymbol{R})\left|3 z^{2}-r^{2}\right| \psi_{0}^{F=1, m_{F}=1}(r, \boldsymbol{R})\right\rangle .
$$

The symmetric Cartesian tensor $\varphi_{i k}(\boldsymbol{R})$ in (9) is given by

$$
\begin{aligned}
\varphi_{i k}(\boldsymbol{R}) & \sim \sum_{\boldsymbol{q}} \mathrm{e}^{\mathrm{i} \boldsymbol{q} \cdot \boldsymbol{R}}\left\{\left\langle R_{S}(r, \boldsymbol{R})\left|V_{\boldsymbol{q}}^{(\mathrm{p})} j_{2}\left(q R_{\mathrm{p}}\right)-V_{\boldsymbol{q}}^{(\mathrm{e})} j_{2}\left(q R_{\mathrm{e}}\right)\right| R_{D}(r, \boldsymbol{R})\right\rangle\right\} \\
& \times\left(3 \frac{q_{i} q_{k}}{q^{2}}-\delta_{i k}\right) .
\end{aligned}
$$

This is the EFG tensor in the centre-of-mass of Ps exciton.

Finally, we have, of course, to average the interaction (9) over the centre-of-mass motion described by the function $\varphi(\boldsymbol{R})$ which is the solution of Eq. (7). We obtain then the effective quadrupole interaction of Ps in noncubic crystals in the form

$$
H_{\mathrm{Q}}=\int|\varphi(\boldsymbol{R})|^{2} H_{\mathrm{Q}}(\boldsymbol{R}) \mathrm{d} \boldsymbol{R}=\left\langle\frac{Q_{\mathrm{Ps}}(\boldsymbol{R})}{4} Q_{i k} \varphi_{i k}(\boldsymbol{R})\right\rangle .
$$

\section{The anisotropy of positronium magnetic quenching}

Let us now analyze the consequences of the effective quadrupole interaction of Ps in a crystal. As well as in the case of $\mathrm{Mu}$ atom [2], this can be easily done by means of the spin-Hamiltonian technique. The corresponding spin Hamiltonian for Ps is anisotropic and given by Eq. (1). It is easy to see that, due to the effective quadrupole interaction, the triplet ground level of such an "anisotropic" positronium is splitted even in the absence of external fields. the form

In the presence of an external magnetic field the Ps spin Hamiltonian takes

$$
H=\omega S_{\mathrm{e}} \cdot S_{\mathrm{p}}-2 \mu_{\mathrm{e}} \boldsymbol{S}_{\mathrm{e}} \cdot \boldsymbol{B}-2 \mu_{\mathrm{p}} \boldsymbol{S}_{\mathrm{p}} \cdot \boldsymbol{B}+\frac{Q_{\mathrm{Ps}}}{4} Q_{i k} \varphi_{i k}-\frac{1}{2} \mathrm{i} \Gamma,
$$

where $\Gamma$ specifies the decay part of the IIamiltonian. Considering the quadrupole term as a perturbation, one can easily obtain the eigenstates and the decay widths of (13):

$$
\begin{aligned}
& E_{0,1}=-\frac{\omega}{4} \mp \frac{\omega}{2} \sqrt{1+x^{2}}-\frac{d}{4} C_{1,0}^{2} \phi(\vartheta, \varphi), \\
& E_{2,3}=\frac{\omega}{4} \pm\left(\mu_{\mathrm{e}}+\mu_{\mathrm{p}}\right) B+\frac{d}{8} \phi(\vartheta, \varphi), \\
& \gamma_{0,1}=\frac{\gamma_{\mathrm{s}, \mathrm{t}}+y^{2}(\vartheta, \varphi) \gamma_{\mathrm{t}, \mathrm{s}}}{1+y^{2}(\vartheta, \varphi)}, \quad \gamma_{2}=\gamma_{3}=\gamma_{\mathrm{t}},
\end{aligned}
$$

where $C_{0,1}=\sqrt{(1 / 2)\left(1 \pm 1 / \sqrt{1+x^{2}}\right)}, y^{2}(\vartheta, \varphi)=y^{2}\left(1+d \phi(\vartheta, \varphi) / 2 \omega \sqrt{1+x^{2}}\right), y=$ $\left(\sqrt{1+x^{2}}-1\right) / x, x=2\left(\mu_{\mathrm{e}}-\mu_{\mathrm{p}}\right) B / \omega, \gamma_{\mathrm{s}}$ and $\gamma_{\mathrm{t}}$ are the decay widths of the singlet 
and the triplet levels of Ps in a medium, $\phi(\vartheta, \varphi)=3 \cos ^{2} \vartheta-1+\eta \sin ^{2} \vartheta \cos 2 \varphi$, $\vartheta$ and $\varphi$ are the polar and the lateral angles characterizing the tilt of $\boldsymbol{B}$ with respect to the principal axis $Z$ of the EFG tensor, $d=Q_{\mathrm{Ps}} \varphi_{z z}, \eta=\left|\left(\varphi_{x x}-\varphi_{y y}\right) / \varphi_{z z}\right|$ is the eflective quadrupole coupling constant of positronium and the asymmetry parameter of the EFG tensor [13], respectively.

As is seen from (14), in the presence of an external magnetic field the effective quadrupole interaction leads to anisotropic splitting of the hyperfine energy levels of Ps and also to anisotropy of its magnetic quenching (see $\gamma_{0,1}$ in (14)). Recall that the application of an external magnetic field on a correlated $\mathrm{e}^{+}-\mathrm{e}^{-}$ pair introduces a partial singlet character in that triplet state of the pair which has no spin component along the direction of the field, and conversely a partial triplet character in its singlet state. This effect was called the magnetic quenching of Ps [3]. In our case the magnetic quenching is anisotropic one with the anisotropy depending on the angle between the $\boldsymbol{B}$ direction and the principle axis $\boldsymbol{Z}$ of the EFG tensor (function $\phi(\vartheta, \varphi)$ inside of $\gamma_{0,1}$ in (14)). In particular, at $\phi(\vartheta, \varphi)=0$, i.e. if $\sin ^{2} \vartheta=2 /(3-\eta \cos 2 \varphi)$ holds (Dean cone), the effective quadrupole interaction does not contribute to the process of magnetic quenching and the magnetic quenching of Ps must here be isotropic. At $\eta=0$ (EFG tensor is axially symmetric) the semivertex angle of the cone is equal to $54^{\circ} 44^{\prime}$. If $\phi(\vartheta, \varphi) \neq 0$ the character of the magnetic quenching of Ps changes due to the effective quadrupole interaction. The processes of quenching differ maximally when magnetic field is parallel and perpendicular to $Z$, the principle axis of the EFG tensor.

It is interesting to estimate the magnitude of the anisotropy of Ps magnetic quenching in a real crystal. The $\alpha$-quartz single crystal seems to be best suited to this purpose. Firstly, the magnitude of crystal field in quartz is small enough [3]. Therefore, we can expect our estimations to be realistic ones. Secondly, this crystal has been investigated well by means of muon spin rotation technique [2]. Therefore, to get the estimations needed we can use Mu data which have been measured experimentally. For example, at $T<70 \mathrm{~K} \mathrm{Mu}$ atom was identified to be localized in the $\alpha$-quartz lattice with the EFG tensor principal axes oriented in the following way: the $\hat{x}$-axis is perpendicular to the twofold crystal axis $\hat{a}$ and makes an angle $\alpha_{0}=(27 \pm 2)^{\circ}$ with the $\hat{c}$-axis, the $\hat{y}$-axis is collinear with the $\hat{a}$-axis and the $\hat{z}$ - and $\hat{c}$-axis make an angle $90^{\circ}-\alpha_{0}$. The EFG tensor is not axially symmetric with $d \approx 9.3 \mathrm{MHz}, \eta \approx 0.35$. At $T>200 \mathrm{~K} \mathrm{Mu}$ moves fast along the $\hat{c}$-axis. As this takes place, the EFG tensor principal axes "rotate" about the $\hat{c}$-axis in such a way that the angle $\alpha_{0}$ remains fixed. Therefore, the effective, a veraged, spin IIamiltonian for $\mathrm{Mu}$ becomes axially symmetric (the axis of symmetry is the $\hat{c}$-axis) with $d \approx-0.5 \mathrm{MHz}, \eta=0$.

Ps in $\alpha$-quartz is observed to be delocalized in a wide temperature range from 88 to $684 \mathrm{~K}[14,15]$. This is similar, to some extent, to the fast $\mathrm{Mu}$ diffusion at elevated temperatures. Therefore, we can expect the spin Hamiltonian for Ps to be axially symmetric as well. The anisotropic splitting of the Ps hyperfine energy levels and the anisotropy of Ps magnetic quehching are given then by Eq. (14) with $\eta=0$. The temperature dependence of the effective quadrupole coupling constant $d$ can be easily estimated in view of the fact that the centre-of-mass wave function 
$\varphi(\boldsymbol{R})$ in Eq. (7) is now of the Bloch wave type

$$
\varphi_{k}(R)=\frac{1}{\sqrt{V}} u_{k}(\boldsymbol{R}) \mathrm{e}^{k \cdot R},
$$

where $u_{k}(\boldsymbol{R})$ is the periodical function of the Ps centre-of-mass coordinate, $k-$ the translational wave vector of $P$ in the crystal. In terms of the wave function (15) Eq. (12) a veraged over the Boltzmann distribution gives us

$$
d(T)=\frac{1}{4 V^{2}}\left(\frac{2 \pi}{M k_{\mathrm{B}} T}\right)^{3 / 2} \sum_{k} \mathrm{e}^{-k^{2} / 2 M k_{\mathrm{B}} T} \int\left|u_{k}(\boldsymbol{R})\right|^{2} Q_{\mathrm{Ps}_{\mathrm{s}}}(\boldsymbol{R}) \bar{\varphi}_{z z}(\boldsymbol{R}) \mathrm{d} \boldsymbol{R} .
$$

Here $\bar{\varphi}_{z z}(\boldsymbol{R})$ denotes $\varphi_{z z}(\boldsymbol{R})$ averaged over the "rotation" of the EFG tensor principal axes, summation is made over the first Brillouin zone. For small $k$ one can approximately put $u_{k}(\boldsymbol{R}) \approx u_{0}(\boldsymbol{R})+\left.\left(\partial u_{k}(\boldsymbol{R}) / \partial k\right)\right|_{k=0} \cdot k$. Then Eq. (16) becomes

$$
\mathrm{d}(T)=A+B \sqrt{T},
$$

where $A$ and $B$ are the functions of the $\alpha$-quartz lattice constants, which may be calculated in an explicit form for a given geometry of an experiment.

Unfortunately, the phenomenological character of the model discussed does not allow one to estimate numerically the magnitude of the anisotropy of Ps magnetic quenching with some degree of certainty. But we will try to do this so as at least to visualize an order of magnitude of the effect. The rough estimations for the magnitude of the anisotropy of Ps magnetic quenching can be obtained for quartz in the following way. In a weak external magnetic field $\left(x^{2}, y^{2}(\vartheta, \varphi) \ll 1\right.$ in Eq. (14)) the magnetic quenching may be characterized by the magnetic quenching parameter [16]. In our case it can be written in the form

$$
Q(\vartheta, \varphi)=y^{2}(\vartheta, \varphi) \frac{\gamma_{\mathrm{s}}}{\gamma_{\mathrm{t}}}
$$

Ifere $y^{2}(\vartheta, \varphi)$ is given by Eq. (14), $\gamma_{\mathrm{s}}=\alpha \gamma_{\mathrm{s}}^{0}+\gamma_{\mathrm{p}}$ and $\gamma_{\mathrm{t}}=\alpha \gamma_{\mathrm{t}}^{0}+\gamma_{\mathrm{p}}$ are the decay widths of the singlet and the triplet Ps in a medium, respectively [3], $\gamma_{\mathrm{s}}^{0}=8 \times 10^{9} \mathrm{~s}^{-1}$ and $\gamma_{\mathrm{t}}^{0}=7.14 \times 10^{6} \mathrm{~s}^{-1}$ are those for Ps atom in vacuum [6], $\alpha$ is the factor taking into account the distortion of the Ps wave function due to the presence of the medium, $\gamma_{p}-$ the Ps pick-off annihilation rate. The relative decrease in the probability of $3 \gamma$-annihilation $(W)$, the enhancement of the narrow component of ACAR (angular correlation of annihilation radiation) curve $(E)$ and the suppression of the long-lived component in the time spectrum $(R)$, measured in the experiments on the magnetic quenching, are defined by the parameter $Q(\vartheta, \varphi)$ as: $W(B)=2 / 3+1 / 3(1+Q(\vartheta, \varphi)), E(B)=Q(\vartheta, \varphi) /(1+$ $Q(\vartheta, \varphi)), R(B)=(1 / 3)\{2+\exp [-Q(\vartheta, \varphi)]\}[16]$. For Ps in $\alpha$-quartz we have $\alpha \approx 1$ [3] and $\gamma_{\mathrm{p}}=0.88 \times 10^{9} \mathrm{~s}^{-1}$ [17]. Then taking into account the conditions $x^{2} \ll 1$ and $\gamma_{\mathrm{t}}^{0} \ll \gamma_{\mathrm{p}} \ll \gamma_{\mathrm{s}}^{0}$ we obtain

$$
Q(\vartheta, \varphi) \approx \frac{x^{2}}{4} \frac{\gamma_{\mathrm{s}}^{0}}{\gamma_{\mathrm{p}}}\left[1+\frac{d \phi(\vartheta, \varphi)}{2 \omega}\right] \approx 1.71 \times 10^{-3}\left[1+\frac{d\left(3 \cos ^{2} \vartheta-1\right)}{2 \omega}\right] B^{2},
$$

where magnetic field is measured in $k^{\prime} G s$.

The magnitude of the parameter $d / \omega$ for Ps in quartz can be estimated using the analogous data for muonium [2]. At room temperatures, when the fast 
Mu diffusion takes place, we have $d^{\mathrm{Mu}} / \omega^{\mathrm{Mu}} \approx-0.12 \times 10^{-3}$. For Ps the $D$-wave admixture forming the quadrupole moment in its ground state (see (3), (8), (10)) is of the order of $\mu_{\mathrm{e}} \mu_{\mathrm{p}}$, whereas for $\mathrm{Mu}$ it is of the order of $\mu_{\mathrm{e}} \mu_{\mu}$. Taking into account the fact that $m_{\mu} \approx 207 m_{\mathrm{e}}$, we obtain $Q_{\mathrm{Ps}} \approx 207 Q_{\mathrm{Mu}}$. If we roughly assume now that the electric field gradient in quartz is the same for delocalized Ps and for fast diffusing $\mathrm{Mu}$, we obtain $d / \omega \approx 4.70 d^{\mathrm{Mu}} / \omega^{\mathrm{Mu}} \approx-0.56 \times 10^{-3}$ (we used the vacuum value for the frequency of the hyperfine splitting of Ps in quartz). As a result, the difference in the values of Ps magnetic quenching parameter for perpendicular and parallel field is equal to

$$
\Delta Q=Q\left(\vartheta=\frac{\pi}{2}\right)-Q(\vartheta=0) \approx 1.44 \times 10^{-6} B^{2} .
$$

For $B \approx 20 \mathrm{kGs}$ we have $\Delta Q \approx 0.06 \%$. Then the differences of the parameters $W$, $E$ and $R$ for parallel and perpendicular magnetic field are equal to $\Delta W \approx 0.02 \%$, $\Delta E \approx 0.06 \%, \Delta R \approx 0.02 \%$, respectively ${ }^{\dagger}$.

\section{Conclusion}

Above we have considered the effective quadrupole interaction of Ps in noncubic defectless crystals. This interaction was shown to lead to the weak anisotropy of Ps magnetic quenching. According to the estimations obtained for delocalized Ps in $\alpha$-quartz, the magnitude of the anisotropy is of the order of one-hundredth fraction of a percent. This corresponds to the small magnitude of crystal field in quartz, on the one hand, but this is evidently too small to observe the effect experimentally, on the other.

Recently the "anomalous" Ps was detected in some polymeric crystalline structures (see [9] and the references therein). Such a Ps is characterized by anomalously small hyperfine splitting (hyperfine splitting is an order of magnitude smaller than that of free Ps in vacuum). Analogous phenomenon is well known for $\mathrm{Mu}^{*}$ atom in some inorganic crystals [18]. Except for anomalously small hyperfine splitting, $\mathrm{Mu}^{*}$ has a very anisotropic hyperfine interaction of the form (1). In this connection it is natural to assume the hyperfine interaction for "anomalous" Ps to be a very anisotropic one as well. Then we can estimate roughly an order of magnitude of the anisotropy of magnetic quenching for "anomalous" Ps in oriented polymeric structures. For this purpose Eq. (19) should be modified with evident substitutions: $\gamma_{\mathbf{s}}^{0} \rightarrow \alpha \gamma_{\mathbf{s}}^{0}, \omega \rightarrow(1 / 3)\left(A_{\|}+2 A_{\perp}\right), d \rightarrow(2 / 3)\left(A_{\|}-A_{\perp}\right)$. For "anomalous" Ps in isotactic polypropylene [9], for example, we have $\alpha=0.55$ and $\gamma_{\mathrm{p}} \approx 0.43 \times 10^{9} \mathrm{~s}^{-1}$. As to the parameter $2\left(A_{\|}-A_{\perp}\right) /\left(A_{\|}+2 A_{\perp}\right)$, for $\mathrm{Mu}^{*}$ in majority of crystals it is $\approx 0.7$ [18]. Using this value for Ps in isotactic polypropylene we obtain from "modified" Eq. (19) $\Delta Q \approx 2 \times 10^{-3} B^{2}$. For $B \approx 10 \mathrm{kGs}$ we have then $\Delta Q \approx 20 \%$. Thus the anisotropic magnetic quenching of "anomalous" Ps in oriented polymeric crystalline structures appears to be observable experimentally.

\footnotetext{
† Using the low temperature $(T<70 \mathrm{~K})$ ratio $d^{\mathrm{Mu}} / \omega^{\mathrm{Mu}} \approx 2.08 \times 10^{-3}$ as a starting point [8], one can get an order of magnitude larger results than those obtained from (20). But the estimations obtained from (20) appear to be more realistic.
} 


\section{References}

[1] V.G. Baryshevskii, S.A. Kuten, Phys. Lett. A 67, 355 (1978).

[2] V.G. Baryshevskii, S.A. Kuten, V.I. Rapoport, Z. Natforsch. A 41, 19 (1986).

[3] A. Dupasquier, in: Positron Solid State Physics, Eds. W. Brandt, A. Dupasquier, Academic Press, New York 1983, p. 510.

[4] T. Hyodo, in: Positron Annihilation, Eds. P.C. Jain, R.M. Singru, K.P. Gopinathan, World Scientific Publ., Singapore 1985, p. 643.

[5] P.J. Schultz, K.J. Lynn, Rev. Mod. Phys. 60, 701 (1988).

[6] V.B. Berestetskii, E.M. Lifshitz, L.P. Pitaevskii, Quantum Electrodynamics, Pergamon, Oxford 1982.

[7] V.G. Baryshevskii, Phys. Status Solidi B 124, 619 (1984).

[8] I.V. Bondarev, S.A. Kuten, Z. Nalforsch. A 49, 439 (1994).

[9] G. Consolati, F. Quasso, J. Phys. C 21, 4143 (1988).

[10] V.I. Goldansky, E.P. Prokopiev, Fiz. Tverd. Tela 13, 2955 (1971).

[11] E.P. Prokopiev, Fiz. Tverd. Tela 19, 472 (1977) (Sov. Phys.-Solid State 19, 271 (1977)).

[12] L.D. Landau, E.M. Lifshitz, Quantum Mechanics. Nonrelativistic Theory, Pergamon, Oxford 1977.

[13] V.S. Grechishkin, Nuclear Quadrupole Interactions in Solids, Nauka, Moskva 1973 (in Russian).

[14] C.II. Hodges, B.T.A. McKee, W. Triftshauser, A.T. Stewart, Can. J. Phys. 50, 103 (1972).

[15] H. Ikari, K. Fujiwara, J. Phys. Soc. Jpn. 46, 92 (1979).

[16] A.Z. Varisov, F.M. Nabiullina, Opt. Spekilrosk. 46, 448 (1979).

[17] A. Greenberger, A.P. Mills Jr., A.T. Thompson, S. Berko, Phys. Lett. A 32, 72 (1970).

[18] B.D. Patterson, Rev. Mod. Phys. 60, 69 (1988). 